IRSTI 29.27.07

https://doi.org/10.26577/phst-2019-2-p2

\title{
Simulation of Coulomb particles collisions and calculation of Lyapunov exponent for bound orbits
}

\author{
K.M. Turekhanova* (iD) and A.M. Murat \\ Institute of Experimental and Theoretical Physics, Al-Farabi Kazakh National University, \\ 71, al-Farabi Ave, 050040, Almaty, Kazakhstan \\ *e-mail: kunduz@physics.kz
}

\begin{abstract}
The computer simulation from the first principles allows us to obtain results for systems consisting of many particles and interacting according to the Coulomb law (plasma, star clusters and astrophysical objects). In this work, the methodology of simulation for Coulomb systems is investigated. The influence of rounding errors, integration errors of reversible Newton equations is studied on the characteristics of Coulomb collisions on the basis of the consideration of several model problems. The trajectories of two particles attracted to the positively charged force center are got for various initial coordinates and velocities and also their energetic natures are researched. It is shown that the symmetries are broken and the particle paths are destroyed due to rounding errors and calculation errors. The degree of deviation from symmetry is described by the value of the Lyapunov exponent, which characterizes the chaos and turbulence in plasmas for the system. The results can be also extended to the system of gravitating masses.
\end{abstract}

Key words: Molecular Dynamics, Coulomb system, elementary processes, Lyapunov exponent, bound orbit, numerical simulation

PACS numbers: 52.20.-j, 52.20.Fs

\section{Introduction}

The physics of elementary processes in dense plasma is one of the fundamental directions in modern physics, $\mathrm{v}$ of such system's properties is of considerable interest in connection with the study of natural plasma phenomena occurring in astrophysical objects, the creation of the scientific foundations of new plasma technologies and the solution of the problem of controlled thermonuclear fusion. There are some problems associated with the lack of adequate theoretical models for some plasma phenomena and the multiparameter nature of such systems. In the experimental study of the properties of such plasmas, In such situations, the problem is partially solved by using modern methods of computer modeling of physical systems [1-7]. The main properties of the classical Coulomb plasma were studied by the method of the dynamics of many particles for determination of the thermodynamic characteristics, the distributed microfields, shielding and etc. [8-12]. In this paper we have used numerical simulation methods for describing of the collisions of Coulomb particles and for estimation of Lyapunov exponent, which characterizes the chaos and turbulence in plasmas. Relaxation of kinetic energy to equilibrium state was studied by molecular dynamic method for nonequilibrium strongly coupled plasmas [13], where the concept of dynamical memory time $t_{m}$ was discussed. The fluctuation of energy $\Delta \mathrm{E}$ and $\mathrm{K}-$ entropy was treated. The Lyapunov exponent in the phase space for ion one-component plasmas with the use of the three-dimensional SCOPE had been evaluated in [9]. The parameter describing chaotic treatment of system was got as a function of the time integral of the correlation function for the second derivative of the interparticle potential, that establishes a link between the Lyapunov exponent and the transport coefficients [14]. There were developed an analytical model for the largest Lyapunov exponent in dilute plasma. The model showed that it related to the dielectric response function [15]. The effect of inevitable experimental noise had been investigated and its full spectrum has been achieved to confirm transition from quasiperiodicity to chaos in plasma [16-18]. 


\section{The simulation method}

The idea of particle method is the numerically solving the motion equations of (Newton's equations) for a system of particles interacting with each other and with the walls. The particle method is based on the ideology of modeling from the first principles (ab initio) [19].

The problem of the occurrence of chaos in dynamical systems also attracts attention in connection with the transition from reversible equations of dynamics to irreversible kinetic equations. The computer simulation from the first principles allows us to obtain results for systems consisting of many particles and interacting according to the Coulomb law (plasma, star clusters). Instability of trajectories, irreversibility of the numerical solutions, mixing of the phase volume in Hamiltonian systems led to a slowdown in recombination in a system of a limited number of particles.

The present work is devoted to the research of the divergence rate of initially close phase trajectories of the classical Coulomb system. In our opinion, the particle method is very fruitful in studying the fundamental properties of a system of many particles. The simulation from the first principles gives the possibility to study the occurrence of chaos in nonlinear dynamical systems. The progress in the study of dynamic chaos based on the Kolmogorov-Arnold-Moser theorem (KAM theory) for the Coulomb system of particles has limited applicability due to the non-integrability of the system [20-22]. In the present work the rate of initially close phase trajectories in the system the plasma non-ideality index, and the calculation accuracy are investigated on the basis of numerical simulation and analytical estimates. For criterion for the similarity characteristics of equilibrium plasma is used the Lyapunov exponent [23-25].

On the calculation of the divergence rate of initially close phase trajectories we use the Lyapunov exponent, which is the most important quantitative characteristic of Hamiltonian and dissipative systems. The Lyapunov exponents determine the measure of stochasticity of Hamiltonian systems [26, 27]. The maximum of them $\sigma_{1}$ is widely used as a criterion for stochasticity [16].

Let the dynamics of a system consisting of $2 n$ particles is determined by the equations:

$$
\frac{d x_{k}}{d t}=V_{k}(x), \quad k=1,2, \ldots, 12 n
$$

The difference between the vectors of two phase trajectories clossing to the initial moment is $\Delta x=x_{2}(t)-x_{1}(t)$, that can be determined from the linearized equation

$$
\frac{d \Delta x}{d t}=G \Delta x, G=\frac{d V}{d x}
$$

where $G$ is the Jacobian matrix. The average speed of the exponential divergence of the initial close phase trajectories is determined as follows $[16,17]$ :

$$
\begin{gathered}
\sigma_{1}=\lim \frac{1}{t} \ln \frac{d(t)}{d(0)} \\
\text { at } t \rightarrow \infty \text { and } d(0) \rightarrow 0
\end{gathered}
$$

where $d(t)=\left|x_{1}(t)-x_{2}(t)\right|=d(0) \exp \left(\sigma_{1} t\right) \quad$ is the distance between close phase trajectories at the initial time $t=0$. The solution of the linear equation for $\Delta x$ can be written as:

$$
\Delta x=T(t) \Delta x(0), T(t)=\exp \left[\int_{0}^{t} G(x(\tau) d \tau)\right]
$$

expanding for small times $\tau$ the matrix exponential $T(\tau)$ in a series we get for eigenvalues of the transition matrix $1+\tau \lambda_{i}$, where $\lambda_{i}$ is eigenvalues of the Jacobian matrix. For a linear system, the maximum eigenvalue $\lambda_{i}$ of the Jacobian matrix determines the rate of exponential recession of the trajectories $\sigma_{1}=\lambda_{1}$. In this case, the path divergence vector becomes collinear to the corresponding eigenvector.

The similarity criterion is the non-ideality parameter $\Gamma=e^{2} /\left(r_{i} T_{e}\right)$, where $r_{i}=\left(3 / 4 \pi N_{i}\right)^{1 / 3}$ is radius of sphere with volume per particle, that follows from the dimension theory for the Coulomb system, where the radius of the sphere with volume per one ion. Therefore, for an equilibrium system with a Maxwellian distribution of particles over velocities, the Lyapunov exponent of particles' system can be represented in the form of 
$\sigma_{1}=t_{c} \sigma_{1}^{0}\left(N_{p}, \Gamma\right)$ where $\sigma_{1}^{0}\left(N_{p}, \Gamma\right)$ is the dimensionless Lyapunov exponent, $t_{c}$ is the characteristic time.

A rough estimate of the Lyapunov exponent for plasma can be obtained from an analogy with the known value of the Lyapunov exponent for rigid spheres with Maxwellian distribution [18]:

$$
\sigma_{1} \approx \frac{V_{0}}{l} \ln \left(1+\frac{l}{2 R}\right)=\frac{3 v_{T} R^{2}}{\pi^{1 / 2} r_{i}^{3}} \ln \left(1+\frac{2 r_{i}^{3}}{3 R^{3}}\right)
$$

where $l=1 / \pi R^{2} N$ is the mean free length, $V_{0}=4 v_{T} / \pi^{1 / 2}$ is the average relative particle velocity, $R$ is the radius of hard spheres, $N$ is their density. The expression (2) determines the increase in the distance between initially close points of the phase trajectories in the form:

$$
d(t)=d(0)[1+l / 2 R]^{V_{0} t / l}
$$

Each collision of particles leads to an increase in the distance between phase trajectories by factor of $1+l /(2 R)$.

\section{The results and discussions}

In presented paper we consider several elementary problems on which there are demonstrated some features of simulation methods of Coulomb collisions. Moreover we acknowledge the effect of calculation errors on the physical result, rounding errors, irreversibility of difference schemes, and to methods for estimating the exponential spread of initially close phase trajectories.

For clarity of presentation we study the dynamics of two particles moving in the same plane in the vicinity of an infinitely heavy (motionless) power center. For convenience there are used the dimensionless units in which the charge and mass of the moving particles are equal to unity.

We consider four formulations of the problems of the motion of two particles in the field of a force center attracting them.

1) The trajectories of two particles attracted to the positively charged force center are showed in figure 1, they repel each other. The initial position of the first particle is a circular orbit of radius 0.05 . The second another particle flies to the first particle from far to the right with an impact parameter of 0.04 on speed 1 . The total energy of the first particle is negative, it is in finite orbit, the total energy of the second particle is positive and it is free, but the full energy of whole system is negative.

The figure obviously shows the physics of the polarization interaction of an electron with a monovalent atom: an electron swooping on an atom polarizes it, an electron begins to be attracted to the induced dipole, then as a result of a close collision it passes to a bound orbit and the first electron becomes free. In this case, the total energy of a free electron after collision increases, i.e. appropriately there is occurred that a bound electron sinks deeper into a potential well. A more detailed study of this process with the calculation of all average characteristics, in particular, the diffusion coefficient of bound electron along the energy axis will be presented in a separate work.

2) The trajectories of two particles, attracted to a positively charged force center are showed in figures 2-4, they repeal each other and their energy characteristics are given in figures 5-8. The initial position of the first particle is the same as in the first problem, but the second is located symmetrically with respect to the center at the same speed. In this formulation, the particles have move along elliptical trajectories symmetrically with respect to the force center.

There are followed the breaking of symmetry and the particle paths are destroyed due to rounding errors and calculation errors. The degree of deviation from symmetry is characterized by the value of the Lyapunov exponent for this system.

3) In figures 9-11 there are demonstrated the trajectories of the same as in the previous problem of the symmetric system, but the particles move in elliptical orbits. The time dependence of the absolute value of the difference in kinetic energy of two charged particles is presented in figures 12, 13 for this system, where they move around the center of force with initial coordinates and velocities.

In this case, the difference in the kinetic energies of the initially symmetric system is determined by the spreading rate of initially close phase trajectories. In this formulation the initial deviation is not specified, but it is obtained as a result of the accumulation of rounding errors over time period in order of 10. Further the divergence of the trajectories (energies) is characterized by exponential growth. The indicator of this growth is the Lyapunov exponent (the slope of the straight line in the semi-logarithmic scale is in figure 13). 


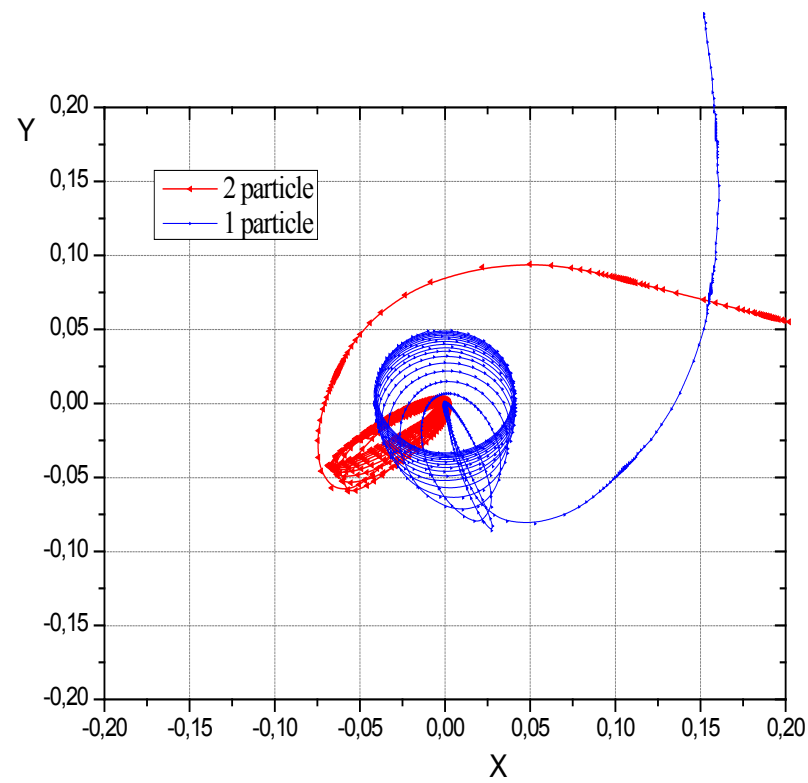

Figure 1 - The trajectory of two charged particles around a force center with initial coordinates and velocities:

$$
\begin{aligned}
& \mathrm{P} 1(0,0.05,0) ; \mathrm{V} 1(-4,0,0) \text { and } \\
& \mathrm{P} 2(1,0.04,0) ; \mathrm{V} 2(-1,0,0)
\end{aligned}
$$

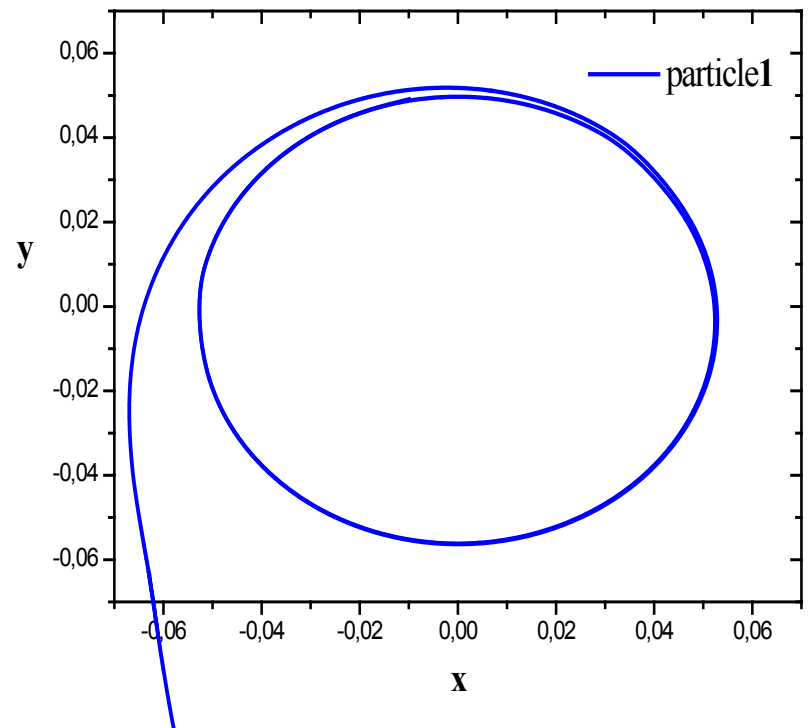

Figure 3 - The trajectory of charged particle around a force center with initial coordinate and velocity:

$$
\mathrm{P} 1(0,0.05,0) ; \mathrm{V} 1(-4,0,0)
$$

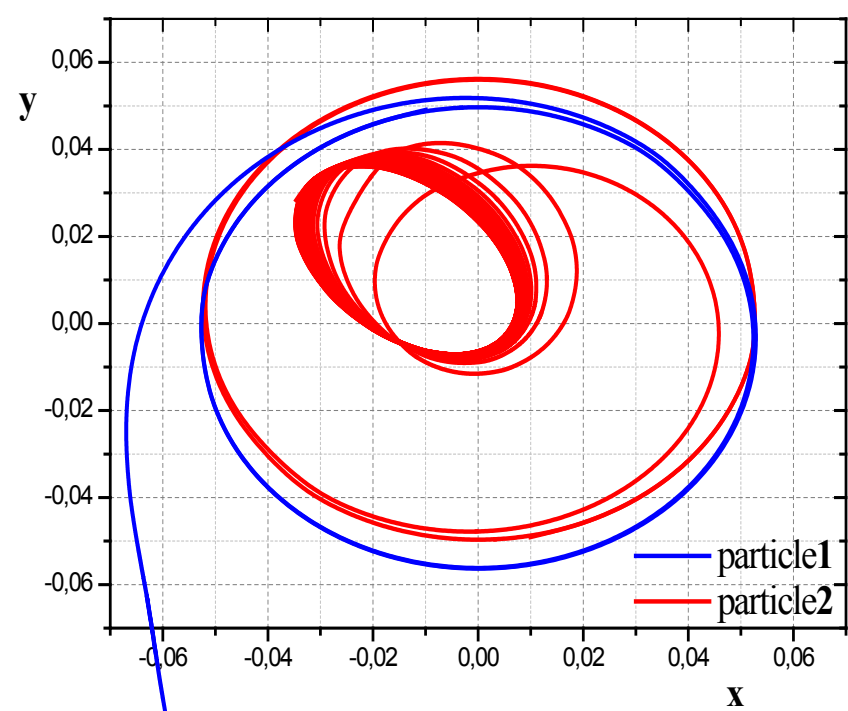

Figure 2 The trajectory of two charged particles around a force center with initial coordinates and velocities:

$\mathrm{P} 1(0,0.05,0) ; \mathrm{V} 1(-4,0,0)$;

$\mathrm{P} 2(0,-0.05,0) ; \mathrm{V} 2(4,0,0)$

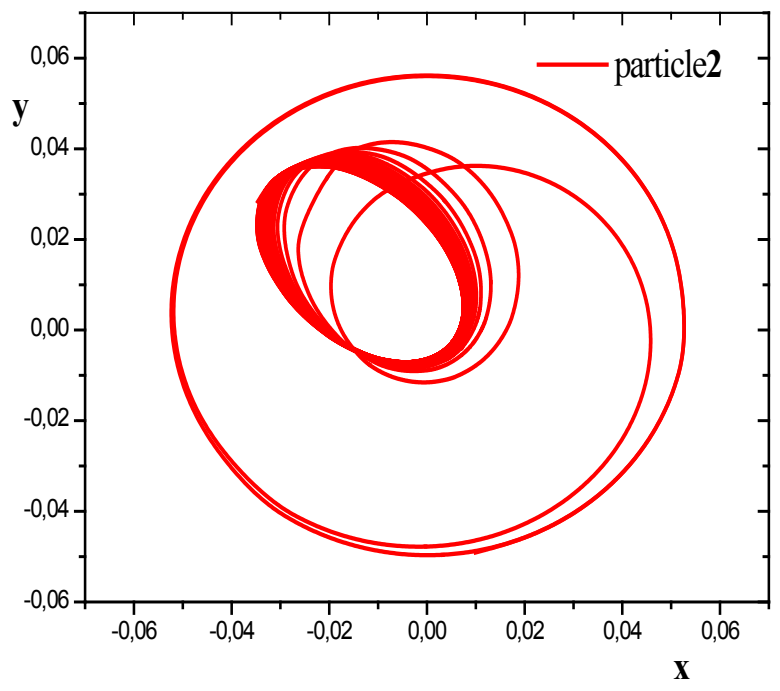

Figure 4 - The trajectory of charged particle around a force center with initial coordinate and velocity: $\mathrm{P} 2(0,-0.05,0) ; \mathrm{V} 2(4,0,0)$ 


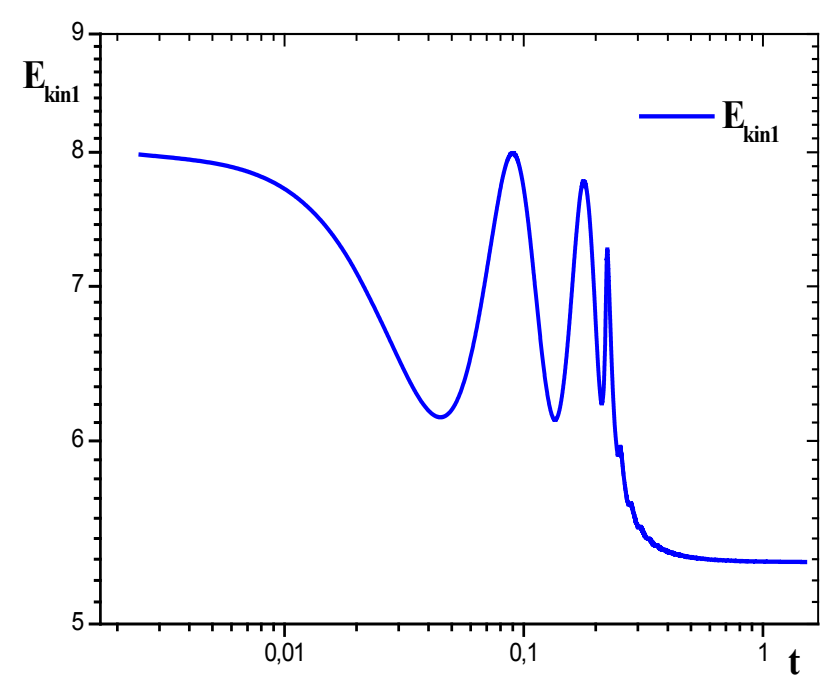

Figure 5 - The kinetic energy of charged particle around a force center with initial coordinate and velocity: $\mathrm{P} 1(0,0.05,0) ; \mathrm{V} 1(-4,0,0)$

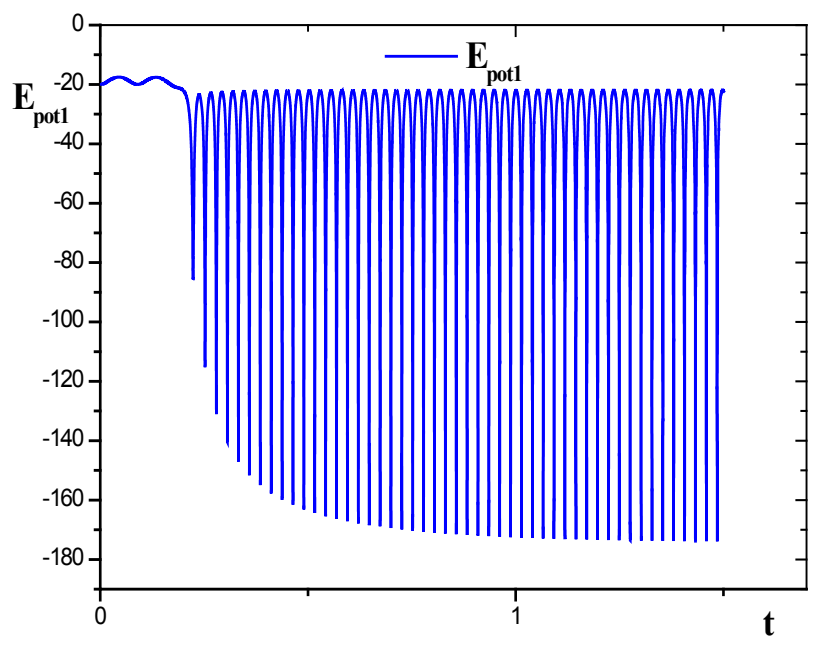

Figure 7 - The potential energy of charged particle around a force center with initial coordinate and velocity: $\mathrm{P} 1(0,0.05,0) ; \mathrm{V} 1(-4,0,0)$

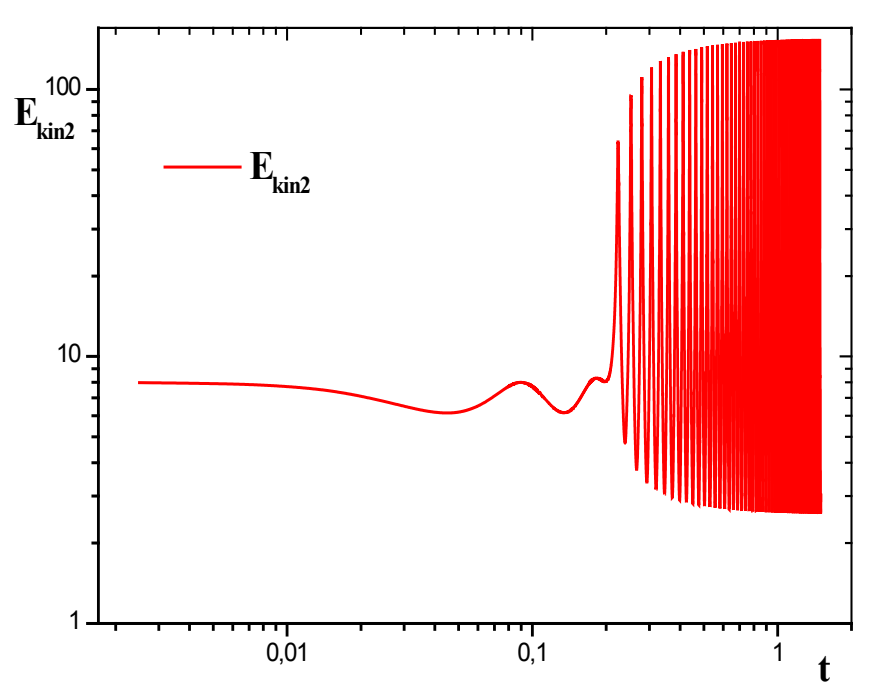

Figure 6 - The kinetic energy of charged particle around a force center with initial coordinate and velocity: $\mathrm{P} 2(0,-0.05,0) ; \mathrm{V} 2(4,0,0)$

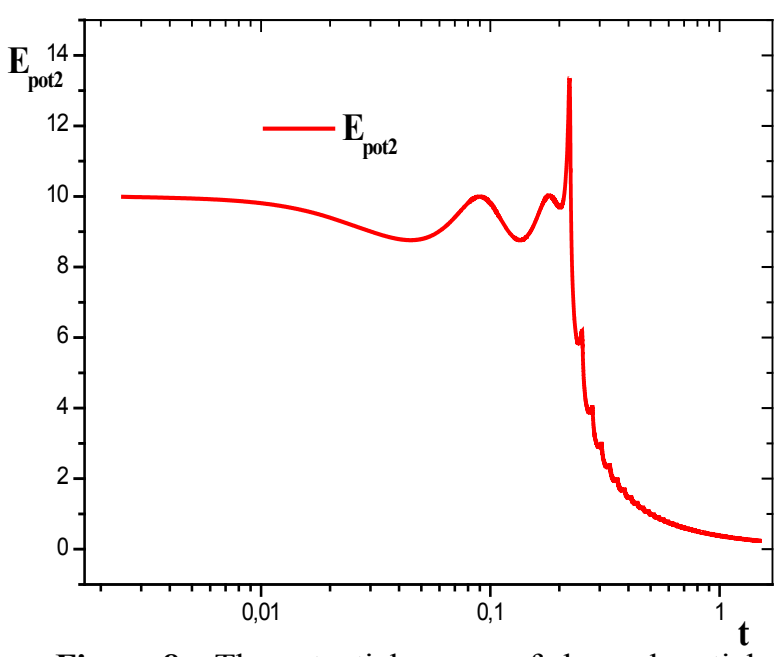

Figure 8 - The potential energy of charged particle around a force center with initial coordinate and velocity: $\mathrm{P} 2(0,-0.05,0) ; \mathrm{V} 2(4,0,0)$ 


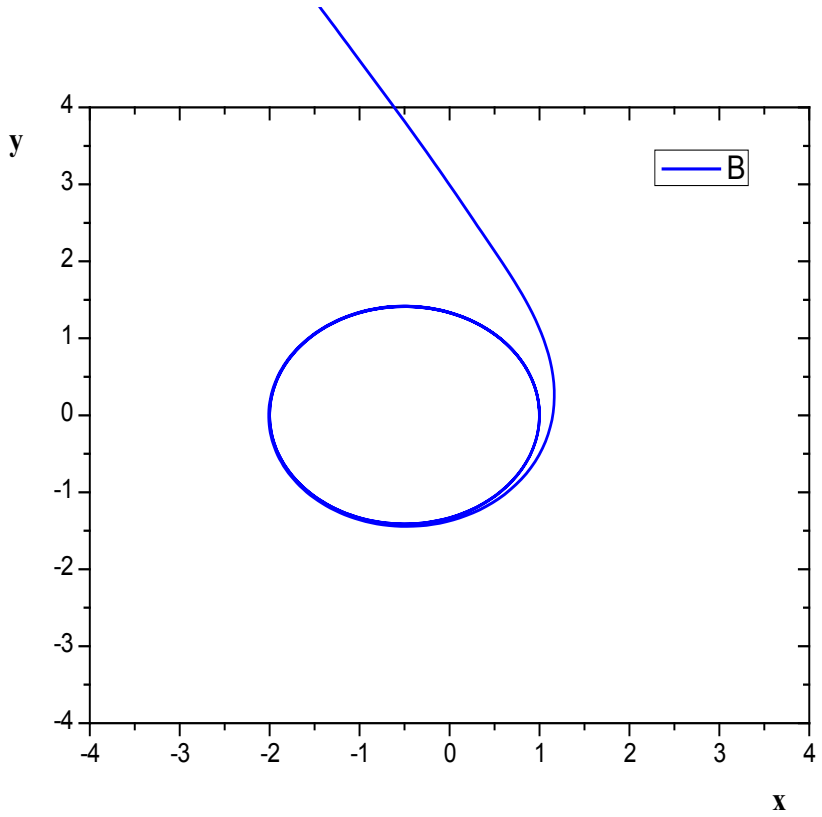

Figure 9 - The trajectory of charged particle around a force center with initial coordinate and velocity: $\mathrm{P} 1(1,0,0) ; \mathrm{V} 1(0,1,0)$

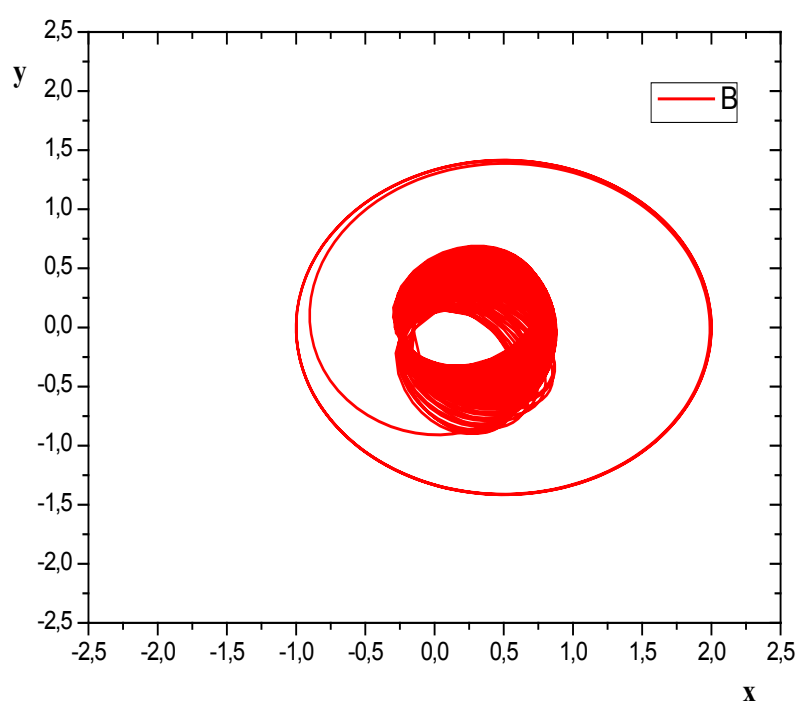

Figure 10 - The trajectory of charged particle around a force center with initial coordinate and velocity: $\mathrm{P} 2(-1,0,0) ; \mathrm{V} 2(0,-1,0)$

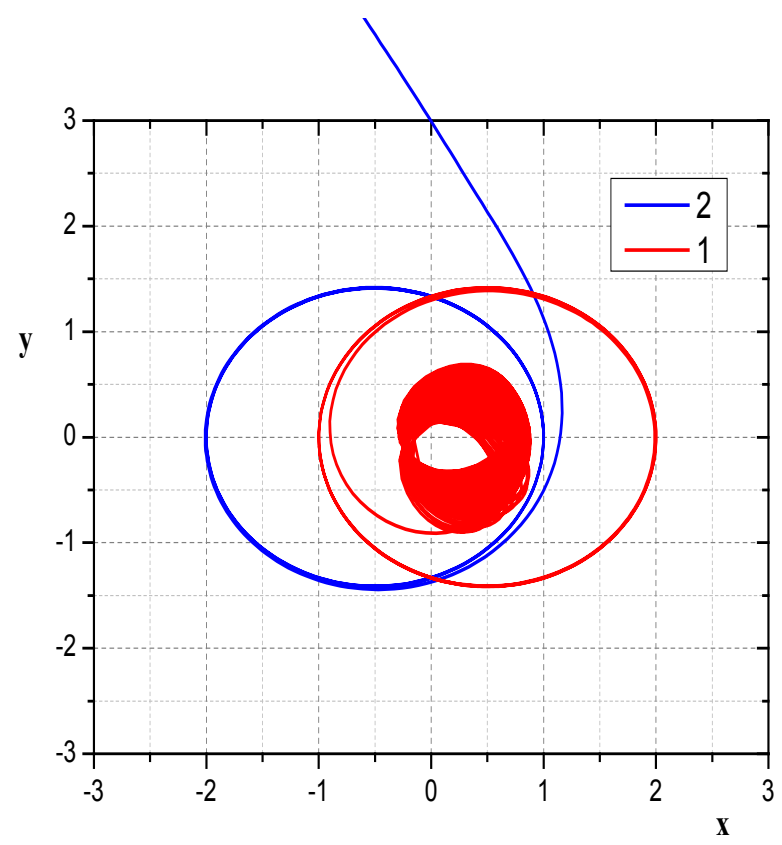

Figure 11 - The trajectory of two charged particles around a force center with initial coordinates and velocities:

$\mathrm{P} 1(1,0,0) ; \mathrm{V} 1(0,1,0)$ and $\mathrm{P} 2(-1,0,0) ; \mathrm{V} 2(0,-1,0)$ 


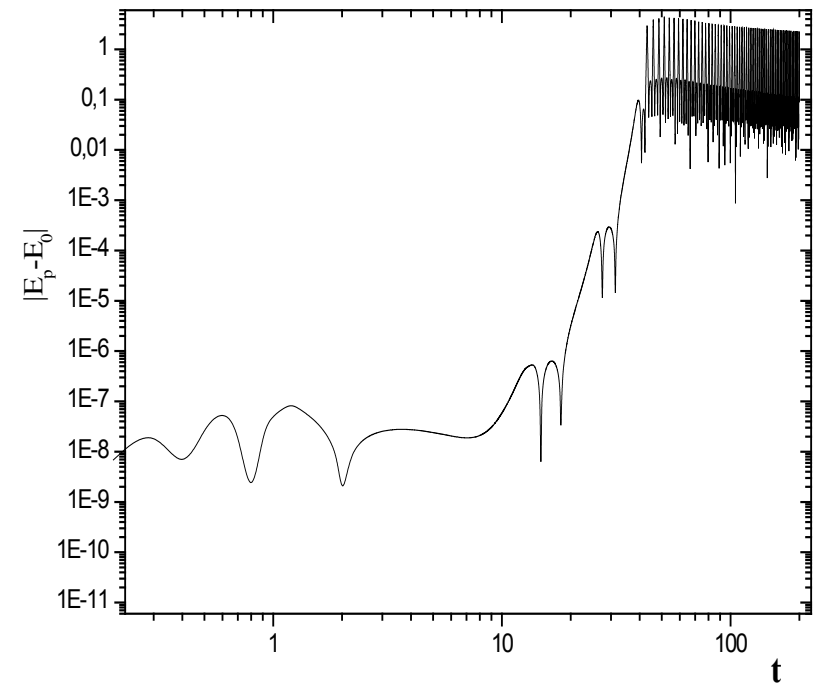

Figure 12 - The time dependence of absolute value of the kinetic energy difference of two charged particles which move around a force center with initial coordinates and velocities:

$\mathrm{P} 1(1,0,0) ; \mathrm{V} 1(0,1,0)$ and $\mathrm{P} 2(-1,0,0) ; \mathrm{V} 2(0,-1,0)$

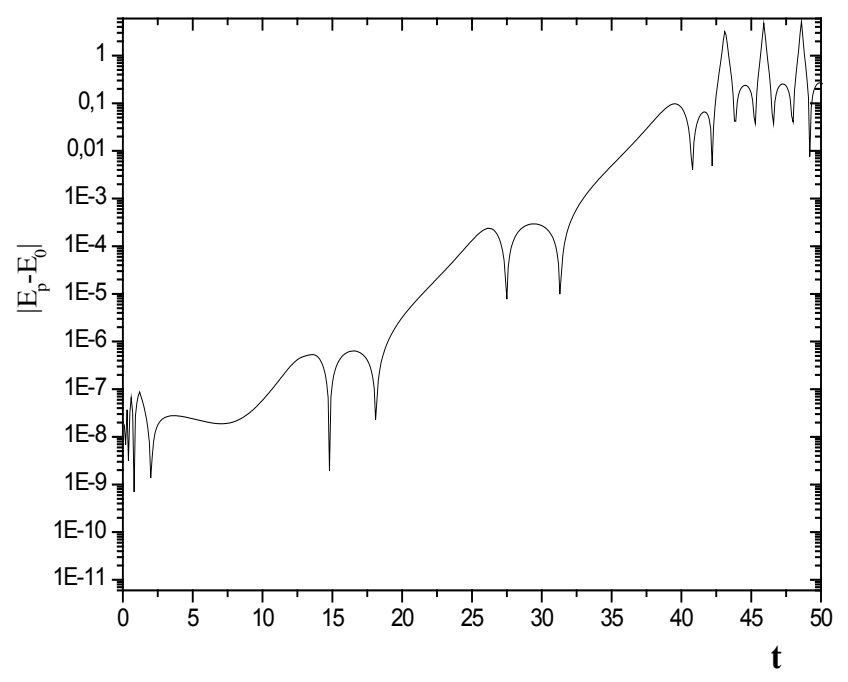

Figure 13 - The time dependence of absolute value of the kinetic energy difference of two charged particles which move around a force center with initial coordinates and velocities:

$\mathrm{P} 1(1,0,0) ; \mathrm{V} 1(0,1,0)$ and $\mathrm{P} 2(-1,0,0) ; \mathrm{V} 2(0,-1,0)$
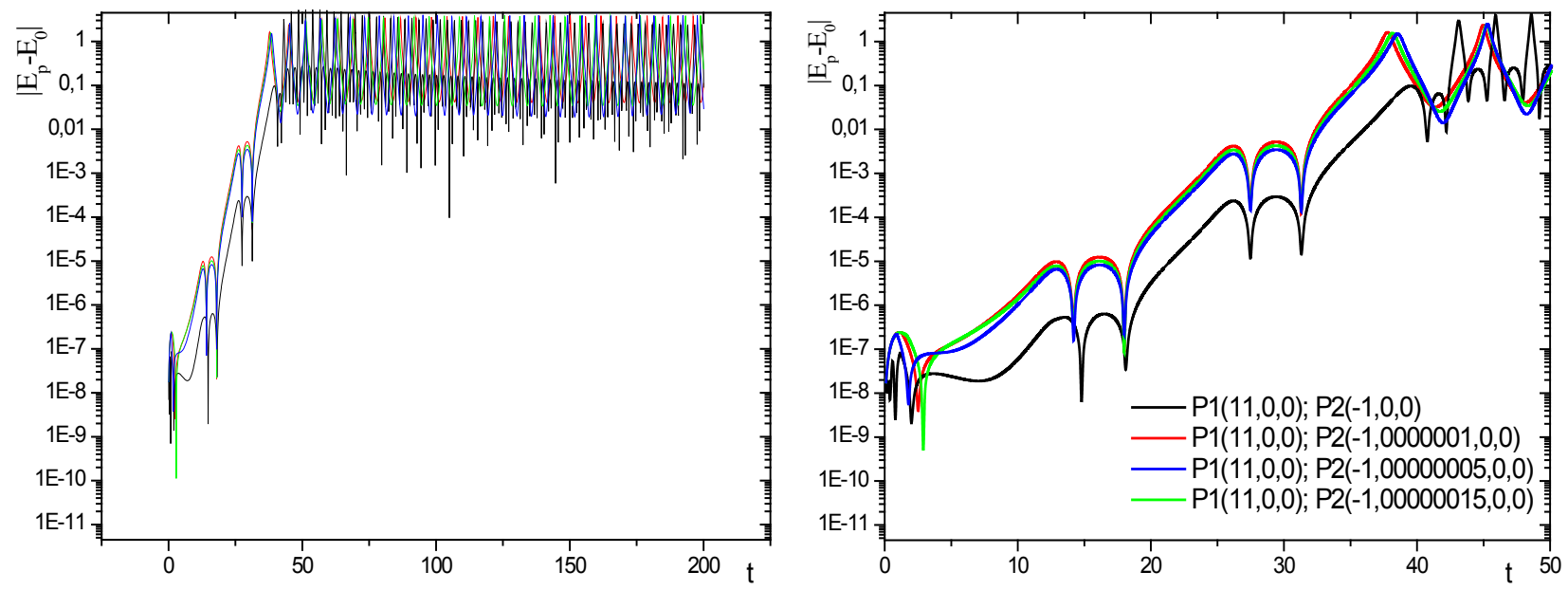

Figure 14 - The absolute value of the change in the difference of kinetic energies of two charged particles around a force center with initial velocities $\mathrm{V} 1(0,1,0)$ и $\mathrm{V} 2(0,-1,0)$ and with different initial coordinates at the initial time

P1 $(11,0,0) ; \mathrm{P} 2(-1,0,0)$

$\mathrm{P} 1(11,0,0) ; \mathrm{P} 2(-1,0000001,0,0)$

$\mathrm{P} 1(11,0,0) ; \mathrm{P} 2(-1,00000005,0,0)$

P1(11,0,0); P2(-1,00000015,0,0) 
4) The similar time-dependent dependences of the absolute value of the kinetic energy difference of two charged particles is showed in figure 14, they move around the center of force with initial coordinates and velocities. But in this case the difference is that the total energies is defined by a bit initial deviation of fluctuation of the second particle's coordinate. In such setting, the further divergence of the trajectories (energies) is also characterized by exponential growth. The indicator of this growth is the Lyapunov exponent (slope of a straight line in a semi-logarithmic scale is in the figure 14).

\section{Conclusions}

In this work the methodology of simulation for Coulomb systems is investigated. The influence of rounding errors, integration errors of reversible
Newton equations is investigated on the characteristics of Coulomb collisions on the basis of the consideration of several model problems. The get results can be also extended to the system of gravitating masses. An estimate of the Lyapunov exponent for system of classical Coulomb particles on dependence of particle's number in the system, the nonideality degree of plasma and the accuracy of integration of Newton's equations can be useful for analyzing the results of modeling systems with a large number of particles. The main attention is paid to the case of the interaction of free and bound particles, which is especially important at consideration of strongly non-ideal systems.

Acknowledgment: The authors would like to thank prof. S.A. Maiorov for helpful discussion. This work was partially supported by the RFBR grant No. 19-08-00611a.

\section{References}

1. Pierleoni C., Margo W.R., Ceperley D.M., \& Bernu B. (1996). Path integral MonteCarlo simulation of hydrogen plasma. In W.D. Kraeft, M. Shlanges (Eds.) . Physics of Strongly Coupled Plasma . London: World Scientific NJ. 11-26.

2. Morozov I.V., Norman G.E., \& Valuev A.A.. (2001). Stochastic properties of strongly coupled plasmas. Phys. Rev. E., 63, 36405.

3. Bezkrovniy V., Filinov V.S., Kremp D., Bonitz M., Schlanges M., Kraeft W.D., Levashov P.R., \& Fortov V.E. ( 2004). Monte-Carlo results for the hydrogen Hugoniot . Phys. Rev. E, 70, 057401

4. Baimbetov F.B., Ramazanov T.S., Dzhumagulova K.N., Kadyrsizov E.R., Petrov O.F., \& Gavrikov A.V. (2006). Modelling of dusty plasma properties by computer simulation methods. J.Phys.A: Math. And Genю, 39, 45214525 . 213029

5. Donkó Z. (2009) . Molecular dynamics simulations of strongly coupled plasmas. J. Phys. A: Math. Theor, 42,

6. Ignatov A.M. (2017). Brownian motion of a plasma crystal . Plasma Physics Reports, 43, 659-667.

7. Chap A.M., \& Sedwick J.R.. (2017). Coulomb collision model for use in nonthermal plasma simulation. Phys. Rev. E, 95, 063209

8. Maiorov S.A., Tkachev A.N., \& Yakovlenko S.I. (1994). Metastable supercooled plasma. Physics-Uspekhi, 37(3), 279-288.

9. Maiorov S.A., Tkachev A.N., \& Yakovlenko S.I. (1995). Metastable state of supercooled plasma. Physics Scripta, 51, 498.

10. Bobrov A.A., Bronin S.Ya., Zelener B.B., Zelener B.V., Manykin E.A., \& Khikhlukha D.R. (2011). Collision recombination coefficient in ultracold plasma. Molecular Dynamics Calculation. JETP, 139, 605.

11. Lankin A.V., \& Norman G.E.. (2009). Collisional recombination in strongly coupled plasma. J. Phys. A, 42, 214032.

12. Bannasch G., \& Pohl T. (2011). Rydberg atom formation in strongly correlated ultracold plasmas. Phys. Rev.A, 84, 052710.

13. Furukawa H., \& Nishihara K. (1990). Reduction in bremsstrahlung emission from hot, dense binary-ionicmixture plasmas. Phys.Rev. A, 42, 3532.

14. Barnett D.M., Tajima T., Nishihara, Ueshima Y., \& Furukawa H. (1996). Lyapunov Exponent of a Many Body System and Its Transport Coefficients. Physical Review Letters, 76, 1812.

15. Nishihara K. \& Ueshima Y. (1999). Lyapunov exponent of dilute gas, liquid and solid plasmas. Plasma Phys. Control. Fusion, 41, A257.

16. Huang W., Ding W. X., Feng D. L., \& Yu C. X. (1994). Estimation of a Lyapunov-exponent soectrum of plasma chaos. Phys. Rev. E, 50. 
17. Ueshima Y., Nishihara K., Barnett D.M., Tajima T., \& Furukawa H. (1997). Particle simulation of Lyapunov exponents in one-component strongly coupled plasmas . Phys. Rev. E, 55, 3439.

18. Pyragas K. (1997). Conditional Lyapunov exponents from time series. Phys. Rev. E, 56, 5183.

19. Hockney, J. R. (1981). Eastwood Computer Simulation Using Particles, New York : McGraw-Hill.

20. Lichtenberg A. J. \& Lieberman M. A. ( 1992). Regular and Stochastic Motion. Springer .

21. Mayer J. \& Goeppert-Mayer M. (1977). Statistical Mechanics. New York: Wiley. 540.

22. Schuster H.G. (1984). Deterministic Chaos. Weinheim: Physik-Verlag.

23. Ebeling W., Kreft W., \& Kremp D. (1977). Theory of Bound States and Ionization Equilibrium in Plasma and Solids. Berlin: Academie-Verlag.

24. Maiorov S.A. (1999). Divergence of the phase trajectories of a Coulomb system. Bulletin of the Lebedev Physics Institute, 1, 25.

25. Eggleston. D.L. ( 2018). Application of chaos theory to the particle dynamics of asymmetry-induced transport. Physics of Plasmas, 25, 032305.

26. Zaslavsky G.M. (1984). Chaos in Dynamical Systems. Moscow: Nauka.

27. Hoover W.G., \& Hoover C.G. (2012). Time Reversibility, Computer Simulation, Algorithms, Chaos (2nd ed) . World Scientific Publishing Company. 